\title{
Challenges and Approaches to Population Management of Long-Term Opioid Therapy Patients
}

\author{
Kari A. Stephens, PhD, Brooke Ike, MPH, Laura-Mae Baldwin, MD, MPH, \\ Christine Packer, MEd, and Michael Parchman, MD, MPH
}

Purpose: Primary care is challenged with safely prescribing opioids for patients with chronic noncancer pain (CNCP), specifically to address risks for overdose, opioid use disorder, and death. We identify sociotechnical challenges, approaches, and recommendations in primary care to effectively track and monitor patients on long-term opioid therapy, a key component for supporting adoption of opioid prescribing guidelines.

Methods: We examined qualitative data (field notes and postintervention interview and focus group transcripts) from 6 rural and rural-serving primary care organizations with 20 clinic locations enrolled in a study evaluating a practice redesign program to improve opioid medication management for CNCP patients. Two independent researchers used content analysis to categorize data into key themes to develop an understanding of sociotechnical factors critical to creating and implementing an approach to tracking and monitoring of patients on long-term opioid therapy in primary care practices.

Results: Four factors were critical to developing a tracking and monitoring system. For each we describe common challenges and approaches used by the clinics to overcome then. The first factor, buy-in and participation, was essential for accomplishing the other 3 . The other factors occurred sequentially: 1) cohort identification-finding the right patients, 2) data collection and extractiontracking the right data, and 3 ) data use-monitoring patients and adjusting care processes.

Conclusions: We identified common challenges and approaches to tracking and monitoring patients using long-term opioid therapy for CNCP in primary care. Based on these findings we provide recommendations to build capacity for tracking and monitoring for organizations that are engaged in improving safe opioid-prescribing practices for CNCP in primary care. (J Am Board Fam Med 2021;34:89-98.)

Keywords: Capacity Building, Chronic Disease, Chronic Pain, Disease Management, Drug Overdose, Focus Groups, Opioids, Primary Health Care

\section{Introduction}

Patients with chronic noncancer pain (CNCP) are often prescribed opioids, potentially increasing their risk for opioid use disorder, overdose, ${ }^{1,2}$ and death. CNCP is 1 of the most frequent presen-

This article was externally peer reviewed.

Submitted 16 March 2019; revised 26 September 2020; accepted 28 September 2020.

From the University of Washington, Seattle, WA (KAS, BI, LMB); Clearwater Valley and St. Mary's Hospital and Clinics, Cottonwood, ID (CP); Kaiser Permanente Washington Health Research Institute, Seattle, WA (MP).

Funding: Grant \# R18HS023750 from the Agency for Healthcare Research and Quality (AHRQ). The contents of this product are solely the responsibility of the author and do not necessarily represent the official views of or imply endorsement by AHRQ or the US Department of Health and Human Services. Additional support was provided by the National Center for Advancing Translational Sciences of the National Institutes of Health under Award Number UL1 TR002319. The content is solely the responsibility of tations in primary care, and the majority of opioid medications are prescribed by primary care providers. ${ }^{3,4}$ The number of patients on long-term opioid therapy (LtOT) within primary care settings increased steadily until 2015, with 3 times the number in 2015 compared with 1999.,

Guidelines for use of LtOT for CNCP care are now available from the Centers for Disease Control and Prevention and others. ${ }^{1,6}$ These guidelines include recommendations for when to initiate, continue, and discontinue LtOT, specifics for selecting and dosing opioids, and processes for assessing risk

\footnotetext{
the authors and does not necessarily represent the official views of the National Institutes of Health.

Conflict of interest: None.

Corresponding author: Kari A. Stephens, PhD, University of Washington, Box 354696, Seattle, WA 98105 (E-mail: kstephen@uw.edu).
} 
of harm from LtOT. Providing guideline concordant care requires that primary care clinics are able to identify and track their patients who use LtOT for CNCP, for example, identifying when patients are due for urine drug testing. Opioid overuse is a patient safety issue that also can be tracked using HIT systems, which often requires discussions and communication among clinical team members on how best to use these information technology systems. ${ }^{7}$ However, little is known about how to best develop and implement effective tracking and monitoring in primary care clinics as they take on initiatives to improve guideline-concordant, evidencebased care for patients with CNCP using LtOT.

The Team-Based Opioid Management study evaluated a team-based approach to practice rede$\operatorname{sign}^{8}$ to improve opioid medication management for patients with CNCP. A key component of the intervention included support for developing tracking and monitoring of guideline-concordant activities, triggers for point-of-care reminders, and monitoring of trends in process and outcome measures at the patient and provider levels. These tracking and monitoring elements were part of a larger intervention targeting comprehensive practice change to adopt safe opioid-prescribing guidelines. Tracking and monitoring requires a socio-technical solution that ensure that both the technical aspects of a tracking and monitoring system and the associated social elements (eg, roles of workforce members in the system) are attended to so that they work smoothly together. A well-established socio-technical model can lead to better development and implementation of health information technology (HIT), ${ }^{9}$ which in turn may influence the success or failure of the practice redesign that the technology is supporting.

In this study we explored the experiences of clinical organizations and the challenges they faced in developing tracking and monitoring systems for patients with CNCP using LtOT. We looked for ways in which the organizations worked on both social and technical elements of the tracking and monitoring systems that they developed. We use our study findings to inform and develop recommendations for building effective tracking and monitoring systems.

\section{Methods}

\section{Intervention, Settings, and Participants}

The Team-Based Opioid Management study provided external support to implement changes in opioid medication management via coaching, clinical education, and provision of relevant resources (eg, model policy and opioid treatment agreement) to 6 rural and rural-serving primary care organizations with 20 clinic locations across Washington State and Idaho. Details of the larger study are provided elsewhere. ${ }^{8}$ Each clinic location had between 2.6 and 7.4 full-time-equivalent clinicians. The organizations were members of the Washington, Wyoming, Alaska, Montana, and Idaho (WWAMI) region Practice and Research Network, a primary care practice-based research network in the 5-state WWAMI region. Five of the organizations included ambulatory care clinics affiliated with critical access hospitals. A total of 2065 patients (range, 165 to 682 in each clinic) met the long-term opioid therapy criteria across the 6 organizations.

Organizations were encouraged to use tracking and monitoring to 1) understand who were the patients receiving long-term opioids, 2) plan for visits, 3) identify care gaps, and 4) monitor overall success of the system-based changes being made through the program. Each organization chose the metrics it wanted to track; there were no study requirements to track specific metrics. Support to the organizations for this work included the option of using an external registry system to track patients on LtOT and/or assistance in developing a sustainable tracking and monitoring approach using internal clinic and organizational resources. All organizations had a local staff member who led data tracking and monitoring during the study. Organizations used 3 different electronic health record (EHR) vendors-Centricity, Epic, and eClinicalWorks.

\section{Data Collection}

Data sources included observational field notes taken by the practice facilitator and clinical advisors during coaching meetings with the organizations ( $\mathrm{n}=108$ meeting notes), and transcripts of 12 semistructured interviews and 11 focus groups with organization leaders, clinicians, and staff at the end of the study. The practice facilitator met 1) quarterly with the organizations' clinicians and staff to discuss practice changes, challenges, ideas for overcoming challenges, and to develop 3 month action plans; and 2) monthly on "shared learning calls" across the organizations. Six interviews were with leadership in the opioid quality improvement teams (1 to 2 participants each), and 6 interviews were with the 
data lead from each of the 6 organizations. Focus group participants included convenience samples of clinicians (from 5 organizations) and staff (from all 6 organizations) in separate groups to ensure each group had freedom to openly discuss challenges to implementation. During interviews and focus groups, we asked participants to tell us their implementation story (including facilitators/barriers, major changes, key lessons, and sustainability plans); they took place over the phone and audiorecordings were transcribed.

\section{Data Coding and Analysis}

Using Dedoose 7.6.21 (Dedoose, Manhattan Beach, CA), 2 analysts individually coded transcripts of the interviews, focus groups, and field notes using the codes "tracking and monitoring," "barrier," "facilitator," and "solution to barrier," meeting regularly to reconcile any coding differences until the codes were applied uniformly. A single coder (BI) then completed coding, adding memoing ${ }^{9}$ throughout to support constant comparisons of emerging themes. Memos and coded excerpts were then analyzed by the core study team (BI and KS) using content analysis ${ }^{9}$ to identify themes related to developing and implementing tracking and monitoring systems for patients on LtOT in primary care practices. These themes were then grouped into 4 factors critical to developing and implementing a tracking and monitoring system, including challenges related to those factors and approaches to overcome these challenges. One analyst (BI) reread the transcripts and field notes to ensure that the analysis results represented the data.
A written summary of the results were presented to participating clinical organization team members. Three of the 6 organizations responded and confirmed that the results represented their experiences, and offered no additional feedback.

\section{Results}

Each organization developed systems with different features to track and monitor patients on LtOT, as shown in Table 1. Organizations 1 and 2 shared an EHR system, and both used a combination of the study-provided external registry and querying of their EHR system for producing monitoring reports. Organization 2 also used an Excel spreadsheet to track the appointments of patients with LtOT, which triggered updates to the external registry. Both organizations were actively exploring using their EHRs to produce tracking and monitoring reports. Organization 3 preferred a simple Excel spreadsheet for tracking and monitoring patients that was not dependent on data from the EHR. Organization 4 attempted to use the studyprovided external registry, but early on chose to move to a proprietary software to pull data out of their EHR, as it felt that this would be a more sustainable model for their organization. Organization 5 had the resources to develop a sophisticated registry system directly linked to their EHR. This organization had a programmer and a quality improvement coordinator who worked with the larger team to develop its registry, train care teams to use it, and then use the system to regularly monitor practices. The care teams themselves could automatically see reports on patients, so no external

Table 1. Description of Tracking and Monitoring System by Clinic Organization

\begin{tabular}{lll}
\hline Organization & Tools Used (Technical) & Data Lead (Responsible for Tracking and Monitoring) \\
\hline 1 & Study registry & MA who oversaw all refills; no other clinical care responsibilities \\
& SHR query reports & \\
& EHR registry & MA with clinical care responsibilities \\
& Excel & \\
& Excel-based registry & \\
3 & Study registry & IT Project Coordinator \\
4 & Proprietary software & Care coordinator (position changed mid-study) \\
5 & EHR integrated registry & Population health data analyst \\
6 & Study registry & Programmer and quality improvement coordinator \\
& Excel & MA with clinical care responsibilities \\
\hline
\end{tabular}

EHR, electronic health record; IT, information technology; MA, medical assistant. 
data lead was necessary. Organization 6 attempted to use the study-provided external registry, but eventually turned to a simple Excel spreadsheet for tracking and monitoring while exploring how to get reports directly from its EHR.

\section{Socio-Technical Factors Critical to Tracking and Monitoring}

We identified 4 factors critical to developing and implementing a tracking and monitoring system for LtOT prescribing. The initial factor, a social culture of engagement and buy-in, was essential for accomplishing the following 3 more technical factors, which occurred sequentially: 1) cohort identification-finding the right patients to track, 2) data collection and extraction-tracking the right data to drive care, and 3) data use-monitoring patients and adjusting practices. Here we describe each of these factors, share challenges inherent in each of these factors, and the approaches organizations took to overcome these challenges.

\section{Factor 1: Culture of Engagement and Buy-In}

The "sociocultural" factor-engagement and buyin across all members of the clinical team, was essential to the success of a tracking and monitoring system. Clinicians and staff collected data from patients, calculated morphine-equivalent doses (MEDs), entered data for easy retrieval for pointof-care and monitoring use, and produced and used reports. Clinics with higher levels of engagement and participation across the practice teams were more likely to succeed in tracking and monitoring their patients on LtOT. Organizations reported that it took active engagement across all levels of the team to get the data needed for tracking and monitoring (eg, providers willing to assign appropriate diagnoses to patients who needed to be tracked, nurses and medical assistants willing to tell the registry manager about new patients).

\section{Challenges}

Organizations encountered significant barriers to gaining buy-in with tracking and monitoring patients on LtOT. Clinicians and staff had concerns that the new protocols would take too much time, given their prior history with EHR technology being time consuming and the high levels of competing demands during their work days. They had difficulty appreciating the potential benefit to their patients to justify the effort.
"I think at first there was a lot of resistance to it because they didn't understand exactly what we were doing."

Organizations also struggled to get buy-in for implementing tracking and monitoring workflows (eg, nurses reminding data leads to update the registry, using an established diagnosis code for LtOT, entering MED totals into the EHR) when the results of these efforts were not clearly beneficial (eg, data reports produced from these efforts were inaccurate or difficult to understand).

"I [data lead] didn't have all the physicians and nursing onboard with giving me the information when a patient came in for their appointment."

Finally, organizations reported that having a clinic champion for the opioid improvement work who reminded staff and providers about the importance of their roles in the tracking and monitoring workflow helped these efforts. Conversely, organizations where champions did not emphasize the importance of the new workflows or did not follow the workflows themselves had a harder time getting others to buy-in to the workflows.

\section{Approaches}

Presenting report data to staff and clinicians in a manner that was useful and easy to understand helped build and maintain buy-in for tracking and monitoring activities. For example, when building reports from the registry, organizations wanted information on data report dashboards to be color coded red, yellow, or green to easily flag areas that needed action. In addition, if the data leads ran reports out of the EHR systems, they would only share the reports once they were cleaned and easier to interpret.

"Make sure patients are coming in green, not yellow on the registry spreadsheet."

Organizations emphasized the importance of verifying data or appropriately framing the purpose of imperfect data before sharing it with clinicians. This developed trust and prevented clinicians from disengaging with the project. Leadership support that clearly messaged the importance of tracking and monitoring activities facilitated meaningful use of the data and led to better buy-in (ie, using the data, helping input data, and helping trigger the need to update data in registries or the EHR; and reporting the belief that tracking and monitoring was useful and important). Organizations found that regular feedback through shared patient panel 
lists, notes, or huddles increased reliance on the tracking and monitoring system and increased buyin for workflows, as expressed by 1 provider:

"I'm glad that [NAME]'s running [the registry] once a month because you think you're floating fine and then you go whoa, how did that happen?"

Organizations that developed clear role definition and dedicated time for staff had the greatest success in tracking and monitoring.

"If you're going to do a registry or do something to track your patients so you know what they're due for, having a resource dedicated to that makes a big difference."

Implementing approaches that integrated multiple clinical team member roles worked well for encouraging participation. For example, an organization that did its data training with staff and clinicians together had a successful launch of their tracking and monitoring program:

"Rolled it out to the providers and MAs at the same time in the same room - that made a really big difference because everyone understood their role."

\section{Factor 2: Cobort Identification}

Organizations first engaged in the tasks of defining and identifying their patients on LtOT, before compiling or collecting specific data related to LtOT treatment.

\section{Challenges}

Organizations did not have uniform solutions for cohort identification. Patients on LtOT lacked an associated label, diagnosis, or single medication that could be easily queried in the EHR, causing organizations to experiment with identification approaches. Their options were technologically limited by what EHR data were present, searchable, and accurate. Organizations with fewer EHR and personnel resources predictably had a harder time with this task. Fifteen months into the observation period, many of the organizations were still refining their approaches. One organization reflected this struggle by saying:

"I think one challenge would be easily identifying these patients... they've got a bistory of opioid use and it's like, how did we miss them?"

\section{Approaches}

Organizations used multiple, varied approaches to overcome challenges in identifying their patients on LtOT. Each approach has strengths and weaknesses, as described in Table 2. The approaches involved searching different data elements (ie, electronic orders for opioid prescriptions, presence of an opioid treatment agreement, MED totals, presence of pain-related diagnoses in combination with prescriptions for opioid medications) and searching different databases such as their local EHRs and registries, and their state's prescription monitoring program databases.

\section{Factor 3: Data Collection and Storage}

After beginning cohort identification, organizations explored how to collect and store opioid management data on each patient for practical use in clinical care and population management. Opioid improvement teams at each organization defined data elements they found helpful to track and monitor to promote guideline recommended care (see Table 3 for the complete list of elements).

\section{Challenges}

Tracking and monitoring all desired data elements was not feasible for most organizations. There were both technical and social reasons that this was not feasible-for example, the EHR did not store data in discrete fields that could be queried or staff/providers did not reliably record data in chart. The 17 data elements spanned a large set of data domains related to providers, encounters, medications, opioid treatment agreements, function/symptom/ screening measures, and lab tests.

"I was surprised bow hard it was to get the data out of our EHR... I was surprised about the data that came out of our EHR. . . how in many cases there were lots of inaccuracies."

Success in collecting and extracting data varied across EHR functionality. Practices struggled with sociocultural and technical challenges, such as getting data entered at the time of appointments because of inadequate staff, difficulty locating data to update their tracking and monitoring system, running reports that included all patients using LtOT, and identifying when patients received care for CNCP during office visits that were not devoted to CNCP. In addition, technical issues prevented some data from being searched or pulled into reports (eg, free text fields, scanned documents, data entered inconsistently), and data that could be pulled into reports were often found to be inaccurate. 
Table 2. Organizational Approaches Used to Overcome Challenges in Identifying Patients on LtOT

\begin{tabular}{|c|c|c|}
\hline Approach & Strengths & Weaknesses \\
\hline $\begin{array}{l}\text { Program using prescriptions to pull } \\
\text { patients into an EHR-linked registry }\end{array}$ & $\begin{array}{l}\text { Can use multiple EHR variables to produce } \\
\text { the registry list and update it efficiently }\end{array}$ & $\begin{array}{l}\text { Required significant IT resources. } \\
\text { Difficult to develop an LtOT definition that } \\
\text { was searchable. } \\
\text { EHR prescription data were sometimes } \\
\text { identified as inaccurate when vetted. }\end{array}$ \\
\hline $\begin{array}{l}\text { Query EHR for patients with opioid } \\
\text { treatment agreement }\end{array}$ & $\begin{array}{l}\text { Organizations frequently prioritized getting } \\
\text { opioid treatment agreements signed as an } \\
\text { early step in improving care }\end{array}$ & $\begin{array}{l}\text { Required developing a custom, searchable } \\
\text { data field for the opioid treatment } \\
\text { agreement if not already present in the } \\
\text { EHR, or doing a chart review. } \\
\text { Sometimes included patients taking other } \\
\text { non-opioid controlled substances. } \\
\text { Missed patients without a signed opioid } \\
\text { treatment agreement. }\end{array}$ \\
\hline $\begin{array}{l}\text { Query EHR for patients with } \\
\text { documentation of MED }\end{array}$ & $\begin{array}{l}\text { Organizations frequently prioritized } \\
\text { calculating MED as an early step in } \\
\text { improving care }\end{array}$ & $\begin{array}{l}\text { Required developing a custom, searchable } \\
\text { data field for MED if not already present } \\
\text { in the EHR, or doing a chart review. } \\
\text { MED often not calculated or } \\
\text { inconsistently calculated. } \\
\text { If MED not updated to } 0 \text { after cessation of } \\
\text { LtOT, over-counted patients. } \\
\text { Assumed MED is calculated only for } \\
\text { patients using LtOT. }\end{array}$ \\
\hline $\begin{array}{l}\text { Query EHR for patients with } \\
\text { prescription for opioid medication }\end{array}$ & $\begin{array}{l}\text { Directly uses the primary element of } \\
\text { interest, opioid prescriptions }\end{array}$ & $\begin{array}{l}\text { Search complicated due to many different } \\
\text { types of opioids, each with many brand } \\
\text { names. } \\
\text { Opioid medication lists require updating as } \\
\text { new opioid medications become available. } \\
\text { Required significant manual cleaning time } \\
\text { to target only patients who were } \\
\text { "currently" receiving LtOT and who met } \\
\text { the definition of LtOT rather than acute } \\
\text { opioid therapy. }\end{array}$ \\
\hline $\begin{array}{l}\text { Query EHR for patients with a } \\
\text { designated diagnosis used to code for } \\
\text { LtOT }\end{array}$ & $\begin{array}{l}\text { Clinician-led cohort identification increased } \\
\text { accuracy of diagnosis }\end{array}$ & $\begin{array}{l}\text { Clinicians resisted applying a designated } \\
\text { diagnosis. } \\
\text { At the time of the study, there was no clear } \\
\text { diagnosis for patients on LtOT. } \\
\text { Relied on care teams knowing how to } \\
\text { consistently apply the diagnosis. }\end{array}$ \\
\hline $\begin{array}{l}\text { Pull provider reports from the state } \\
\text { prescription monitoring database }\end{array}$ & Useful cross-check of internal data & $\begin{array}{l}\text { Organizations thought the state drug } \\
\text { database lists were inaccurate. } \\
\text { Was not possible to run a clinic-wide } \\
\text { report, required running individual } \\
\text { provider reports. } \\
\text { Required manual cleaning time to identify } \\
\text { only those patients who met the } \\
\text { definition of LtOT rather than acute } \\
\text { opioid therapy. }\end{array}$ \\
\hline
\end{tabular}

EHR, electronic health record; IT, information technology; LtOT, long-term opioid therapy; MED, morphine-equivalent dose.

\section{Approaches}

Organizations started addressing these challenges by prioritizing 1 or 2 data elements based on feasibility and priority, and then expanded to more data elements as they were able. Generally, MED was prioritized as an initial variable to track, as it was immediately actionable and meaningful to providers.

"This patient is like this $X$ amount [MED], everybody's eyes bulged... it's somebody that's been here for years... just knowing how much or what that number is, it just kind of surprises the providers."

To track and monitor MED, organizations focused on developing consistent approaches to calculating and storing MED, which required technical problem solving such as putting the same MED calculator on every desktop and creating a queryable MED field in the EHR, and socio- problem solving, such as training staff. 
Table 3. Data Elements Identified by Organizations for Monitoring LtOT Patients

Data Element

Prescriber

Date of last appointment

Date of next appointment

Diagnosis to identify patients on LtOT

MED

Co-prescription of opioids and sedatives

Date opioid treatment agreement signed

Function assessment (e.g., PEG)

Risk assessment (e.g., ORT)

Depression assessment (e.g., PHQ)

Date of last state prescription monitoring database check

Result of last state prescription monitoring database check

Date of last urine drug test

Result of last urine drug test

Sleep apnea assessment (e.g., STOPBang)

PTSD assessment

Anxiety assessment (e.g., GAD-7)

GAD-7, General Anxiety Disorder - 7; MED, morphine-equivalent dose; ORT, opioid risk tool; PEG, Pain, Enjoyment, and General activity pain assessment tool; PHQ, patient health questionnaire; PTSD, post-traumatic stress disorder.

Practices experimented with 4 different strategies to collecting and storing patient data (Table 4). Strategies tended to hinge on both technical and personnel-based resources at the clinic and included EHR-linked registries, Excel spreadsheets, proprietary software, and native HER-querying functi-onality. Training staff and providers was an essential step to ensure usable data were collected, no matter which approach was used.

"We had to ask them to be very specific on bow they entered what it was so that we could pull it out properly."

\section{Factor 4: Data Use}

After identifying patients and collecting and extracting pertinent data elements, organizations began to plan and experiment with ways to use the data. The ways that organizations used their tracking and monitoring systems included 1) ensuring quality patient care by identifying care gaps and appropriately planning for visits, and 2) monitoring overall success and clinical variation in managing the population of patients on LtOT. Two organizations targeted all these uses and 4 organizations targeted a subset of uses.

\section{Challenges}

Ability to access the data and create reports with actionable information for their clinicians and staff limited some clinics' use of the data. Organizations with access to data struggled with both technical and sociocultural challenges to ensure data validity, optimally organize and report the data so they would be well received and be easy to use, and embed use of the data into clinic workflows and processes.

\section{Approaches}

The following approaches represent the ways that organizations were able to use their data within the constraints of the aforementioned challenges.

Data Use 1: Ensuring Quality Patient Care by Identifying Care Gaps and Appropriately Planning for Visits and Refills. Five of the 6 organizations improved how they used data for visit planning, including identifying patients on LtOT with upcoming appointments, identifying which care gaps needed to be addressed, and providing a summary of the patient's status for the care team. Having these data easily accessible to staff and providers streamlined processes to help ensure patients received a service when needed, as described by 1 clinic manager:

"So now our staff can look in and say when their last UDT (urine drug test) was and they get it collected even before the provider goes in. So, I think for standardizing things, it's made it much easier for the practice and for the staff."

Three organizations had opioid improvement teams that reviewed patient reports to check for care gaps and identify high-risk patients. The tracking and monitoring manager would leave notes about needed care processes in patient charts or provide patient reports for huddles, so that care teams could create plans to close care gaps and attend to high risk patients.

"[The registry allows us to be] better in touch with who those at-risk patients are and how they're being managed."

"We bave a better idea of what's going on with our patients so we can really concentrate on safety, their safety, and making sure that we're providing the best care that we can."

Data Use 2: Monitoring Overall Success and Clinical Variation. Five organizations used reports to monitor whether they were successful in meeting their opioid improvement goals. Organizations did not set goals until they determined availability of data, what was feasible to report, and reviewed those baseline metrics. Organizations noted that sharing the overall monitoring reports with clinicians and staff not only motivated them to continue 
Table 4. Socio-Technical Strategies for Storing and Accessing LtOT Patient-Related Data

\begin{tabular}{|c|c|c|c|}
\hline Strategy & Strengths & Weaknesses & Quote \\
\hline EHR-linked registry & $\begin{array}{l}\text { Data extracted directly from the } \\
\text { EHR into the registry. } \\
\text { Easy to access detailed reports. }\end{array}$ & $\begin{array}{l}\text { Required significant resources to } \\
\text { build (time, skills, funding). } \\
\text { Clinicians had to click out of } \\
\text { the EHR to reach the registry. }\end{array}$ & $\begin{array}{l}\text { "Once the data is there and the } \\
\text { structure is built, the work is } \\
\text { just finding the data. A lot of } \\
\text { the work was collaborative } \\
\text { with the folks who work with } \\
\text { the EHR. Digging and finding } \\
\text { where the template data was } \\
\text { stored." }\end{array}$ \\
\hline Excel spreadsheet & $\begin{array}{l}\text { Easy to control (change } \\
\text { variables, edit entries, track } \\
\text { elements for updating). } \\
\text { Inexpensive. } \\
\text { Provided an interim system until } \\
\text { an EHR-integrated system } \\
\text { was possible }\end{array}$ & $\begin{array}{l}\text { Required manual chart review or } \\
\text { data entry by clinical } \\
\text { personnel (e.g., medical } \\
\text { assistant) to populate with } \\
\text { data. } \\
\text { Hand-entered data from chart } \\
\text { reviews onto excel spreadsheet } \\
\text { (errors more likely, time } \\
\text { consuming). } \\
\text { Needed a cue to know when } \\
\text { there were new data to enter. } \\
\text { Cumbersome to keep } \\
\text { historical data, therefore } \\
\text { difficult to track trends. } \\
\text { Required IT support to make } \\
\text { more usable (e.g., turning the } \\
\text { font red when patient overdue } \\
\text { for a urine drug test). } \\
\text { Not integrated with the EHR } \\
\text { for use in patient care. }\end{array}$ & $\begin{array}{l}\text { Excel is a "quick and easy } \\
\text { reference." } \\
\text { "If I was gone or something, I } \\
\text { would miss getting the flags } \\
\text { from the nurses saying that } \\
\text { hey, we refilled this } \\
\text { medication. So I never really } \\
\text { took the time to go and } \\
\text { backtrack, I just went forward } \\
\text { from there." }\end{array}$ \\
\hline Proprietary software & $\begin{array}{l}\text { Data from the EHR extracted } \\
\text { with proprietary software into } \\
\text { a report. } \\
\text { Simple to use, others could step } \\
\text { in with minimal training. }\end{array}$ & $\begin{array}{l}\text { Not all proprietary software in } \\
\text { use at organizations was } \\
\text { nimble enough to easily create } \\
\text { LtOT reports. } \\
\text { Proprietary software still needed } \\
\text { a list of patients to query, } \\
\text { which required maintenance. }\end{array}$ & $\begin{array}{l}\text { "They're adding COT module, } \\
\text { but they haven't done that yet; } \\
\text { we're already married up to } \\
\text { them and we like it, but they } \\
\text { aren't there yet." }\end{array}$ \\
\hline EHR query & $\begin{array}{l}\text { Data extracted directly from the } \\
\text { EHR. } \\
\text { Did not require additional } \\
\text { system or proprietary } \\
\text { software. }\end{array}$ & $\begin{array}{l}\text { Required translation of query } \\
\text { into a tracking report. } \\
\text { Required double-checking via } \\
\text { chart review or provider } \\
\text { consultation as reports often } \\
\text { included errors. } \\
\text { Difficult to troubleshoot why } \\
\text { errors occurred. } \\
\text { Difficult to limit to current } \\
\text { patients on LtOT.Exporting } \\
\text { from EHR to Excel produced } \\
\text { a report that took hours to } \\
\text { make readable. }\end{array}$ & $\begin{array}{l}\text { "Inquiries in [EHR] are pretty } \\
\text { primal; created several of our } \\
\text { own, but they're fraught with } \\
\text { problems; we never get the } \\
\text { same list of patients." } \\
\text { "Exporting is a pain... It does } \\
\text { not produce a usable } \\
\text { spreadsheet-it takes hours to } \\
\text { go through it to make it } \\
\text { usable." }\end{array}$ \\
\hline
\end{tabular}

COT, chronic opioid therapy; EHR, electronic health record; IT, information technology; LtOT, long-term opioid therapy.

to improve, but also helped identify challenges to address.

"Having data is part of the psychology. Becomes synergistic with buy-in when clinicians and staff can see the data change."

Five organizations used reports to look for clinical variation. Opioid improvement teams identified when a clinician was practicing outside of the clinic's policies and met 1-on-one with the clinician to offer assistance. One opioid improvement member noted:
"After providers hear about 3 or 4 times on the same patient, they realize they need to do something about it."

Reports were also reviewed at regular medical staff meetings, which facilitated conversations about how to handle complex patients, informed clinicians of their prescribing patterns compared with others, and created peer pressure as a motivator to adhere to agreed-on practices. Leadership felt that these conversations built the buy-in needed to drive 
process changes and helped providers and staff feel supported by the larger clinical team in implementing the work.

"We showed [the report] to them in the fall and they were very competitive... 'I only have [this many patients] over the 120-[MED] mark, bow many do you have?"”

Organizations tended to be most successful in monitoring their opioid improvement initiatives when they started with simple approaches to identify, track, and monitor patients using existing resources that built on past experience, and then developed more complex approaches over time.

\section{Discussion}

Tracking and monitoring of patients on LtOT required a socio-technical approach, ${ }^{9}$ one that combines technical solutions with social processes. The 4 factors we identified as critical to developing a tracking and monitoring system overlap comprehensively with the dimensions of Sittig and Singh's ${ }^{10}$ socio-technical model that have been noted to drive successful development and implementation of HIT solutions. ${ }^{9}$ Namely, our 4 factors illustrate various aspects of each domain described by Sittig and Singh ${ }^{10}$ : hardware and software, clinical context, human computer interface, people, workflow and communication, internal organizational features, and measurement and monitoring. In addition, clinics used a variety of flexible approaches to overcome challenges, which highlights the importance of tailoring tracking and monitoring approaches to available technical and personnel-related resources. The varied approaches used by the organizations in this study provide a rich source of tailoring options for clinical organizations engaged in improving their care for patients on LtOT.

Universal challenges included generating buyin from clinicians and staff for the effort of tracking and monitoring, accessing data from EHRs in efficient and accurate ways, and identifying relevant patients to track. Inefficiencies and inaccuracies of technical approaches based on the EHR meant the personnel resources needed for tasks such as identifying the patient population on LtOT were substantially greater than initially anticipated and difficult to sustain. Clinics with less leadership buy-in had greater difficulty succeeding at practice change, regardless of their chosen approaches.

\section{Specific Recommendations for Tracking and Monitoring Patients on LtOT in Primary Care}

Based on the lessons learned from organizations in this study, we make the following recommendations to practices establishing tracking and monitoring systems for patients on LtOT:

1. Assess your organization's current tracking and monitoring processes for patients on LtOT and the resources available for the effort. Practices in our study chose to use and augment their existing tracking systems for efficiency as they had limited personnel and technical resources available. Assessing these resources upfront to set achievable goals early in the process may be helpful.

2. Consider a variety of approaches to identify the cohort of patients on LtOT, tailor to your organization's resources, and fine-tune the approach over time. Be clear with clinicians and staff that it is a "work in progress" and will improve over time. Practices in our study reported that they worked with their providers and staff in an iterative fashion to build reliable lists of patients on LtOT over time.

3. Select a limited set of initial data elements for tracking and reporting to staff and providers. Focus on getting those data elements accurately and consistently collected, starting simple and expanding to more comprehensive data collection as able. Practices in our study selected a priority set of data elements to begin tracking, rather than trying to track all of them from the beginning.

4. Make sure that organizational leaders prioritize this work, set aside protected time for data management, and foster buy-in among clinicians and staff by making time for reflective conversation, conveying the importance to patient safety, and defining clear roles and responsibilities. Practices in our study noted challenges with shifting personnel roles and responsibilities and ensuring that personnel were available to do the tracking and monitoring.

5. Identify feasible approaches to using data for planning visits and refills, monitoring clinicians' practices, and measuring success. Be consistent, not perfect. Practices in our study noted that monitoring just a couple elements (e.g., MED, co-prescribing) was doable for the organization and useful for monitoring quality care. 
6. Share data with leadership, clinicians, and staff regularly to stimulate the ongoing conversations necessary to create buy-in for tracking and monitoring as a means to improving care. Practices noted that clinician and staff buy-in was key to success; sharing data that convey the value of tracking the vulnerable population of patients on LtOT can help reinforce or grow buy-in over time.

\section{Conclusion}

We identified both sociocultural and technical challenges and approaches to tracking and monitoring patients using LtOT for CNCP in primary care. Organizations had varying levels of success in tracking and monitoring their patients. Strong leadership and team buy-in, having established or easy-to-use data tracking tools, and using progressive strategies moving from simple to more complex approaches tended to drive success. All these organizations iterated solutions to develop customized approaches based on current resources and environment. Based on our findings, we make a set of recommendations to build capacity for tracking and monitoring to drive improvements in opioid-prescribing practices.

To see this article online, please go to: http://jabfm.org/content/ 34/1/89.full.

\section{References}

1. Dowell D, Haegerich TM, Chou R. CDC guideline for prescribing opioids for chronic pain-United States. MMWR Recomm Rep 2016;65:RR1-R49.
2. Chou R, Fanciullo GJ, Fine PG, et al. Clinical guidelines for the use of chronic opioid therapy in chronic noncancer pain. J Pain 2009;10:113-30.

3. Mansfield KE, Sim J, Croft P, Jordan KP. Identifying patients with chronic widespread pain in primary care. Pain 2017;158:110-9.

4. Mansfield KE, Sim J, Jordan JL, Jordan KP. A systematic review and meta-analysis of the prevalence of chronic widespread pain in the general population. Pain 2016;157:55-64.

5. Dunn KM, Saunders KW, Rutter CM, et al. Opioid prescriptions for chronic pain and overdose: a cohort study. Ann Intern Med 2010;152:85-92.

6. Washington State Agency Medical Directors' Group. Interagency guideline on prescribing opioids for pain. 3rd ed. 2015. Available from: http://agencymeddi rectors.wa.gov/Files/2015AMDGOpioidGuideline. pdf.

7. Lanham HJ, Leykum LK, McDaniel RR, Jr. Same organization, same electronic health records (EHRs) system, different use: exploring the linkage between practice member communication patterns and EHR use patterns in an ambulatory care setting. J Am Med Informat Assoc 2012;19:382-91.

8. Parchman ML, Penfold RB, Ike B, et al. Teambased clinic redesign of opioid medication management in primary care: effect on opioid prescribing. Ann Fam Med 2019;17:319-25.

9. Birks M, Chapman Y, Francis K. Memoing in qualitative research: probing data and processes. J Res Nurs 2008;13:68-75.

10. Sittig DF, Singh H. A new sociotechnical model for studying health information technology in complex adaptive healthcare systems. Qual Saf Health Care 2010;19:i68-i74. 